\title{
Plutonium : modèle de calcul des doses engagées*
}

\author{
P.G. BEAU** \\ (Manuscrit reçu le 18 octobre 1983)
}

\begin{abstract}
RÉSUMÉ
Dans sa publication 30 , la Commission internationale de protection radiologique (CIPR) propose des limites annuelles d'incorporation pour un certain nombre de radionucléides. Ces limites sont basées sur le calcul de doses engagées qui comporte deux parties : l'une métabolique, l'autre dosimétrique, les deux faisant appel à des modèles. L'exploitation de ces modèles est présentée en vue de l'évaluation des doses engagées dans le cas d'une contamination interne par inhalation d'oxyde de plutonium. On a cherché également à montrer l'influence des caractéristiques physiques et physicochimiques du composé radioactif sur la dose engagée par les variations qu'elles entraînent pour certains paramètres des modèles, en particulier ceux liés au métabolisme.
\end{abstract}

\section{ABSTRACT}

In its publication ICRP 30 , the International commission on radiological protection presents annual limits of intake for a number of radionuclides. These limits are based on the calculation of the committed doses, which includes two parts : a metabolic one and a dosimetric one, both using models. The utilization of these models is presented in the perspective of evaluating the committed doses in the case of internal exposure by inhalation of plutonium oxide. The effect of the physical and physico-chemical properties of the radioactive compound on the committed dose is also demonstrated as they result in variations of some model parameters, especially those related to metabolism.

\section{INTRODUCTION}

Cet article vise à préciser et à exploiter les concepts et les formulations présentées par la Commission internationale de protection radiologique (CIPR) dans sa publication 30. Le but de cette dernière est de donner pour un certain nombre de radionucléides des limites d'incorporation annuelles. L'obtention de ces valeurs est basée sur le calcul de doses engagées dont les étapes figurent de manière dispersée dans l'ouvrage et ses annexes

* Cet article reprend des éléments de la communication présentée aux Journées d'études SFRP “Plutonium et Radioprotection”, Saclay, 14-16 juin 1983.

** Commissariat à l'énergie atomique, Institut de protection et de sûreté nucléaire, Département de protection sanitaire, BP 6, 92260 Fontenay-aux-Roses. 
si l'on se propose de considérer un radionucléide particulier. En prenant l'oxyde de plutonium comme exemple, nous avons essayé de présenter ces étapes dans un ordre logique en précisant certains points d'ordre métabolique ou dosimétrique. On a également fait jouer la possibilité de faire varier certains paramètres intéressant le métabolisme de l'aérosol inhalé.

\section{I.1. Notion de dose engagée}

La notion de dose engagée repose sur le fait que les radionucléides ne délivrent pas, dès leur incorporation, comme le ferait une source externe de rayonnement pénétrant, la totalité de leur énergie à l'organe ou tisșu pour lequel l'élément stable a, s'il existe, une affinité physico-chimique.

En effet, les radionucléides sont soumis simultanément à des phénomènes de transfert à partir des portes d'entrées vers les organes et d'élimination de ces organes en même temps qu'ils subissent leur propre décroissance radioactive. II en résulte une évolution du débit de dose au cours du temps jusqu'à la décroissance effective totale. Il est donc indispensable de connaître le temps de séjour des radionucléides dans les organes en terme de période effective et cela est d'autant plus important que la période physique est longue, ce qui est le cas du plutonium. La décroissance effective totale ne survenant pour des radionucléides de ce type qu'après des temps très longs, il s'est avéré que l'intégration du débit de dose devait être faite sur une durée correspondant pour les travailleurs à la période d'emploi, soit au maximum 50 années. La dose absorbée résultant de cette intégration pour un apport unique de radionucléide est la dose engagée. La dose engagée par unité d'activité incorporée est une quantité constante. Dans le cas d'apports successifs, elle est applicable à chacun d'eux. La dose engagée correspond au comportement du radionucléide non modifié par une intervention à caractère thérapeutique. Formulée mathématiquement, la dose engagée pour un organe ou tissu T répond à l'expression :

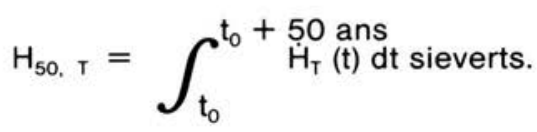

où $\dot{H}_{\mathrm{T}}(\mathrm{t})$ est le débit d'équivalent de dose à l'organe considéré.

\subsection{Débit d'équivalent de dose en exposition interne}

Le débit d'équivalent de dose $\dot{H}_{T}(t)$ s'exprime par le produit de deux quantités :

$\dot{H}_{T}(t)=S\left(\varepsilon_{T}\right) \cdot r_{T}(t) \frac{S v}{j}$

La première, $S\left(\varepsilon_{\mathrm{T}}\right)$, que nous nommerons facteur dosimétrique, réunit les paramètres biophysiques de l'irradiation. La seconde, $r_{T}(t)$, appelée fonction de rétention effective, rassemble les paramètres décrivant le comportement métabolique du radionucléide dans l'organe $\mathrm{T}$. 


\subsubsection{Facteur dosimétrique $S\left(\boldsymbol{\varepsilon}_{T}\right)$}

Il est décomposable, à son tour, en un produit de deux termes :

Un terme purement dosimétrique de conversion de l'énergie en dose absorbée :

$\mathrm{k}=1,602 \times 10^{-10} \frac{\mathrm{g} \cdot \mathrm{Gy}}{\mathrm{MeV}}$

Un terme appelé énergie effective spécifique qui tient compte du fait que les organes qui ont concentré le radionucléide constituent des volumes sources $S$ qui nécessitent que leur soit appliqué un modèle d'absorption d'énergie fonction du caractère pénétrant ou non du rayonnement. A ces volumes sources sont associés des volumes cibles $T$ qui sont les autres organes susceptibles d'être irradiés par les organes sources, ces derniers étant leur propre cible. Dans ces conditions, l'énergie effective spécifique est, pour un couple organe source $\mathrm{S}$ - organe cible T, l'énergie moyenne absorbée par gramme d'organe cible pour un rayonnement de type i. En symbolisant cette énergie par $\varepsilon(T \leftarrow S)_{i} \frac{M e V}{\text { dés.g }}$ on a : $\mathrm{S}\left(\boldsymbol{\varepsilon}_{\mathrm{T}}\right)_{\mathrm{i}}=k . \boldsymbol{\varepsilon}(\mathrm{T} \leftarrow \mathrm{S})_{\mathrm{i}} \frac{\mathrm{Sv}}{\text { dés }}$

le facteur de qualité du rayonnement étant inclus dans les formulations de $\varepsilon(T \leftarrow S)_{i}$.

Une autre quantité sans dimension incluse dans la formulation précédente est appelée fraction absorbée FA $(T \leftarrow S)_{i}$ et traduit le modèle d'absorption d'énergie. Dans le cas des rayonnements non pénétrants tels que les alphas du plutonium où l'on fait l'hypothèse que les énergies sont complètement absorbées dans l'organe source on a pour :

$\mathrm{S}=\mathrm{T} \quad \mathrm{FA}(\mathrm{T} \leftarrow \mathrm{T})=1$

$S \neq T \quad F A(T \leftarrow S)=0$

$\sum_{i} \varepsilon(T-S)_{i}$ est tabulée pour un radionucléide déterminé dans la publication 30 de la CIPR.

\subsubsection{Fonction de rétention effective $r_{T}(t)$}

Elle décrit au niveau d'un organe ou tissu $\mathrm{T}$ la combinaison des phénomènes d'élimination biologique et de décroissance radioactive. Elle se définit comme le rapport de la quantité présente au temps t, à celle présente au temps zéro. La notion de rétention effective recouvre, en fait, plusieurs situations biologiques, ce qui a conduit à distinguer :

- la rétention à la porte d'entrée qui reflète le comportement du radionucléide au niveau des portes d'entrées respiratoire et digestive en fonction des modèles anatomophysiologiques correspondants ;

- la rétention élémentaire qui reflète le comportement du radionucléide à partir d'une activité introduite directement dans le sang. Le radionucléide se distribue dans un ou plusieurs organes pour lesquels joue, s'il existe, l'affinité physico-chimique propre à l'élément stable ; 
- la rétention après incorporation qui prend en compte l'ensemble des phénomènes cinétiques de la porte d'entrée à l'organe inclus.

\section{DOSES ENGAGÉES DANS LE CAS DU PLUTONIUM}

Pour étudier les doses engagées dans le cas du plutonium, l'exemple du ${ }^{239} \mathrm{PuO}_{2}$ a été retenu parce qu'il s'agit d'une forme physico-chimique bien connue et fréquemment rencontrée. C'est aussi une forme inhalable mettant en œuvre tous les mécanismes de dépôt et d'épuration respiratoire. Elle conduit également à une diffusion sanguine après inhalation avec une répartition du radionucléide entre le foie et le squelette principalement. La possibilité d'une incorporation par ingestion, théoriquement envisageable, présente un intérêt limité en milieu professionnel et ne donne lieu qu'à une très faible diffusion systémique. L'analyse sera donc centrée sur l'inhalation.

\section{II.1. Dose engagée à l'appareil respiratoire}

\section{II.1.1. Modèle de dépôt et d'épuration respiratoire}

L'appareil respiratoire constitue à la fois une voie de pénétration de l'aérosol de ${ }^{239} \mathrm{PuO}_{2}$ dans l'organisme et un organe soumis au risque par l'irradiation dont il est l'objet. Le dépôt des particules inhalées se fait par le jeu de phénomènes purement physiques qui sont fortement influencés par la taille particulaire. II a été constaté que les valeurs du dépôt correspondant à chacunes des régions nasopharyngée (NP), trachéobronchique (TB) et pulmonaire $(P)$ de l'appareil respiratoire varient en fonction du diamètre aérodynamique médian en masse (DAMM) ou en activité (DAMA) des particules dont la taille suit une distribution statistique.

Un modèle de dépôt a été établi par la CIPR: il donne la variation des fractions déposées $D_{\mathrm{NP}}, D_{\mathrm{TB}}, \mathrm{D}_{\mathrm{P}}$ d'une activité inhalée unité en fonction du paramètre de taille précédent. La figure 1 montre que ces variations sont importantes.

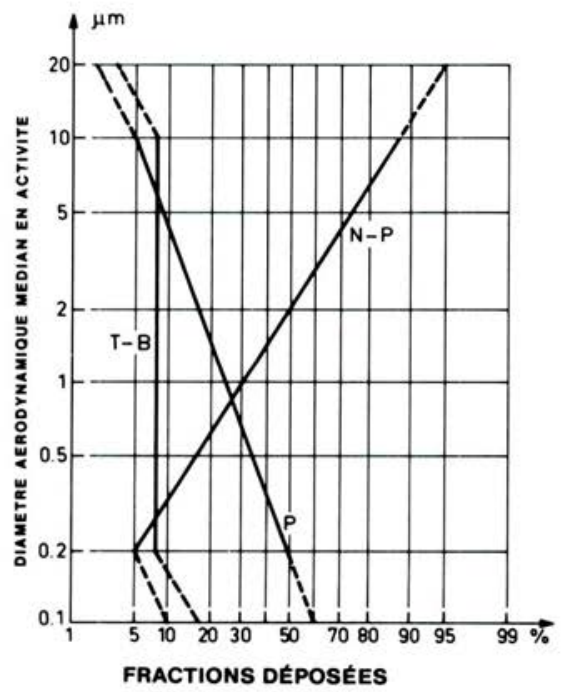

Fig. 1. 
Les particules déposées sont mobilisées par un système d'épuration qui assure leur transfert par des phénomènes biologiques et physicochimiques vers le tube digestif et vers le sang circulant et les liquides extracellulaires.

Un modèle d'épuration complète, par conséquent, le modèle de dépôt. La modélisation de ces transferts a recours à la représentation compartimentale (fig. 2). Les compartiments NP, TB et $P$ sont divisés en souscompartiments : 2 pour NP et TB, 4 pour $P$. Ces sous-compartiments correspondent à une fraction $f$ du dépôt s'éliminant à un taux fixe $\lambda(j-1)$ par les voies $a, b . .$. i vers les compartiments sanguin et digestif. Un compartiment lymphatique $L$ est placé en dérivation entre le sous-compartiment $h$ et le compartiment sanguin. Trois classes d'épuration ont été définies en fonction de la vitesse d'élimination des composés radioactifs commune aux sous-compartiments $\mathrm{e}, \mathrm{g}, \mathrm{h}$. $\mathrm{PuO}_{2}$ appartient à la classe $\mathrm{Y}$ d'épuration lente.

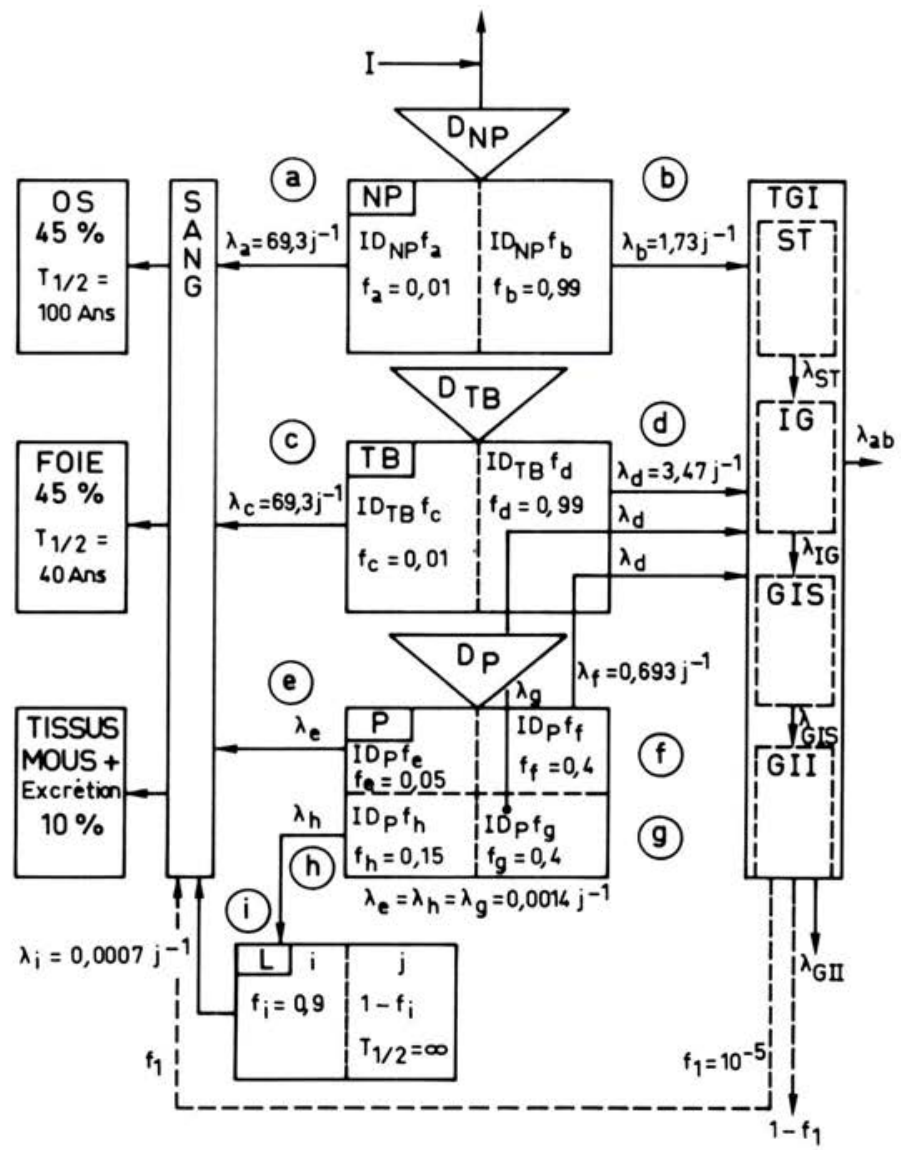

Fig. 2. - Schéma métabolique pour ${ }^{239} \mathrm{PuO}_{2}$ - classe $\mathrm{Y}$. 
II.1.2. Formulation de la rétention à partir du modèle pour $\mathrm{PuO}_{2}$ (classe $Y$ )

La structure du modèle conduit à une formulation de la rétention par compartiment. Le compartiment NP n'est pas pris en compte en raison des périodes biologiques très courtes associées aux sous-compartiments a et b. Au total, pour l'ensemble de l'appareil respiratoire (RESP) on a la somme :

$r_{\text {RESP }}(t)=r^{T B}(t)+r^{P}(t)+r^{L}(t)$

\section{Région TB}

A ce niveau, la rétention effective répond à la somme :

$$
r^{\mathrm{TB}}(\mathrm{t})=\underbrace{r_{\mathrm{c}}^{\mathrm{TB}}(\mathrm{t})}_{\begin{array}{c}
\text { transfert } \\
\text { sanguin }
\end{array}}+\underbrace{r_{\mathrm{d}}^{\mathrm{TB}}(\mathrm{t})+r_{\mathrm{f}}^{\mathrm{TB}}(\mathrm{t})+r_{g}^{\mathrm{TB}}(\mathrm{t})}_{\begin{array}{c}
\text { transfert } \\
\text { digestif }
\end{array}}
$$

Chaque rétention composante correspond à la solution d'une ou plusieurs équations différentielles du $1^{\text {er }}$ ordre à coefficients constants qui décrivent le cinétique de transfert du radionucléide ou de son composé à partir d'un des sous-compartiments concernés (c, $d, f, g)$.

\section{Transfert sanguin}

L'évolution de l'activité $Q_{c}$ est réglée par l'équation différentielle :

$\frac{\mathrm{d} Q_{\mathrm{c}}}{\mathrm{dt}}=-\lambda_{\mathrm{c}} \mathrm{Q}_{\mathrm{c}}$ dont la solution est :

$Q_{c}(t)=Q_{c}(0) e^{-\lambda_{c} t}$

Les conditions initiales sont fournies immédiatement par le modèle : $Q_{c}(0)=I D_{T_{B}} f_{c}$ où I est l'activité inhalée

d'où $Q_{C}(t)=I D_{T B} f_{C} e^{\lambda_{c} t}$

et la rétention effective

$$
\begin{aligned}
r_{c}^{T B}(t) & =\frac{Q_{c}(t)}{I} e^{-\lambda_{R} t}=D_{T B} f_{c} e^{-\left(\lambda_{C}+\lambda_{R}\right) t} \\
& e^{-\lambda_{R} t} \text { étant l'exponentielle de décroissance radioactive. }
\end{aligned}
$$

\section{Transfert digestif}

Pour la rétention composante relative à la fraction $f_{d}$ de l'aérosol déposé en TB, la démarche est la même que pour la fraction $f_{c}$. On a :

$$
\begin{aligned}
& Q_{d}(t)=I D_{T B} f_{d} e^{-\lambda_{d} t} \\
& r_{d}^{T B}(t)=D_{T B} f_{d} e^{-\left(\lambda_{d}+\lambda_{R}\right) t}
\end{aligned}
$$


Pour les deux autres composantes la situation est plus compliquée. Elles correspondent au passage en TB des particules radioactives déposées en $P$, mobilisées par les voies $f$ et $g$, et finalement éliminées par la voie $d$. Cette situation est décrite au plan cinétique par un système couplé de deux équations différentielles. On le formulera seulement pour la voie f, la voie $\mathrm{g}$ donnant lieu à une écriture identique :

$$
\begin{aligned}
& \frac{d Q_{f}}{d t}=-\lambda_{4} Q_{f} \\
& \frac{d Q_{d}}{d t}=+\lambda_{4} Q_{f}-\lambda_{d} Q_{d}
\end{aligned}
$$

La première équation différentielle donne immédiatement :

$Q_{f}(t)=I D_{p} f_{f} e^{-\lambda_{f} t}$

En portant cette solution dans la deuxième équation différentielle on a :

$$
\frac{d Q_{d}}{d t}=+\lambda_{f} I D_{p} f_{f} e^{-\lambda_{f} t}-\lambda_{d} Q_{d}
$$

dont la solution est :

$Q_{d}(t)=\frac{\lambda_{1} I D_{p} f_{f}}{\lambda_{d}-\lambda_{t}}\left[e^{-\lambda_{t} t}-e^{-\lambda_{d} t}\right]$

et la rétention effective correspondante :

$$
r_{f}^{T B}(t)=\frac{\lambda_{t} D_{p} f_{f}}{\lambda_{d}-\lambda_{f}}\left[e^{-\lambda_{f} t}-e^{-\lambda_{d} t}\right] e^{-\lambda_{R} t}
$$

Cette expression, somme de deux fonctions exponentielles décroissantes, l'une positive, l'autre négative, et de mêmes conditions initiales, est connue sous le nom de fonction de Bateman. Cette fonction donne à la courbe de rétention une forme en dôme plus ou moins aplati selon la valeur des constantes en exposant des deux exponentielles. Le fait que les exposants sont les constantes de transfert du modèle est caractéristique de la cinétique par transfert irréversible entre compartiments en chaîne.

\section{Région P}

Pour cette région on a l'expression :

$$
\begin{aligned}
& r^{P}(t)=\underbrace{r_{e}{ }^{P}(t)}+\underbrace{r_{h}{ }^{P}(t)}_{v}+\underbrace{r_{f}^{P}(t)+r_{g}{ }^{P}(t)} \\
& \text { direct indi- transfert } \\
& \text { rect digestif } \\
& \underbrace{\text { par L }}_{\text {transfert }} \\
& \text { sanguin }
\end{aligned}
$$


Chacune des composantes de la rétention est régie par une équation différentielle du type précisé pour la partie transfert sanguin en TB, l'ensemble des solutions conduisant à l'expression :

$r^{p}(t)=D_{p}\left[f_{f} e^{-\lambda f^{t}}+\left(f_{e}+f_{g}+f_{h}\right) e^{-\lambda} p^{t}\right] e^{-\lambda R^{t}}$

où $\lambda_{\mathrm{p}}$ est la constante d'épuration commune aux trois voies $\mathrm{e}, \mathrm{g}, \mathrm{h}$.

\section{Région L}

On trouve dans cette région une disposition en chaîne pour les souscompartiments $h$ et $i$ qui conduit à une fonction de Bateman pour la rétention effective :

$r_{i}^{L}(t)=\frac{\lambda_{p} D_{p} f_{h} f_{i}}{\lambda_{i}-\lambda_{p}}\left[e^{-\lambda} p^{t}-e^{-\lambda_{i} t}\right] e^{-\lambda_{R} t}$

Cette rétention ne décrit le comportement que de la partie mobilisable du compartiment L. Une partie non mobilisable correspond au sous-compartiment $\mathrm{j}$ qui ne joue biologiquement qu'un rôle de réservoir et n'est donc soumis qu'à la décroissance radioactive.

Avec $\mathrm{f}_{\mathrm{i}}=1-\mathrm{f}_{\mathrm{i}}$ et $\lambda_{\mathrm{i}}=0$, on $\mathrm{a}$ :

$r_{i}^{L}(t)=\frac{\lambda_{p} D_{p} f_{h}\left(1-f_{i}\right)}{\lambda_{p}}\left[1-e^{-\lambda} p^{t}\right] e^{-\lambda R^{t}}$

et au total :

$r^{L}(t)=r_{i}^{L}(t)+r_{j}^{L}(t)$

\subsubsection{Formulation de la dose engagée}

Le débit de dose à l'appareil respiratoire répond en fonction des données précédentes à l'expression :

$\dot{\mathrm{H}}_{\text {RESP }}(\mathrm{t})=\mathrm{S}\left(\boldsymbol{\varepsilon}_{\mathrm{RESP}}\right)_{\alpha} \cdot \mathrm{r}_{\mathrm{RESP}}(\mathrm{t})$

où $\mathrm{S}\left(\boldsymbol{\varepsilon}_{\mathrm{RESP}}\right)_{\alpha}$ est obtenue pour une énergie $\alpha$ de $5,15 \mathrm{MeV}$ et une masse de l'organe RESP de $1000 \mathrm{~g}$. L'intégration sur 50 ans va porter sur les rétentions composantes et permettre de calculer les quantités $U$ utilisées dans la publication 30 :

$\mathrm{U}_{50, \text { TB }}^{\mathrm{r}}=\mathrm{K} \cdot \int_{0}^{\mathrm{T}} \mathrm{r}^{\mathrm{TB}}(\mathrm{t}) \mathrm{dt} \frac{\text { désintégrations }}{\mathrm{Bq} \text { inhalé }}$

$U_{50, P}^{Y}=K \cdot \int_{0}^{T} r^{P}(t) d t \quad \quad \quad \quad$

$U_{50, L}^{Y}=K \cdot \int_{0}^{T} r^{L}(t) d t \quad " \quad "$ 
$T$ est le nombre de jours en 50 ans (18250), et le facteur $\mathrm{K}$ égal à 86400 secondes par jour assure la conversion des becquerels . jours en becquerels. secondes pour donner le nombre total de transformations nucléaires (désintégrations) dans l'organe. La dose engagée est :

$\mathrm{H}_{50, \text { RESP }}^{Y}=\mathrm{S}\left(\varepsilon_{\mathrm{RESP}}\right)_{\alpha} \cdot U_{50, \text { RESP }}^{Y} \frac{\mathrm{Sv}}{\mathrm{Bq} \text { inhalé }}$

avec $U_{50, \text { RESP }}^{Y}=U_{50, T B}^{Y}+U_{50, P}^{Y}+U_{50, L}^{Y}$

\section{II.1.4. Importance de la granulométrie}

Les valeurs de la dose à l'appareil respiratoire qui figurent habituellement dans les publications pour $1 \mathrm{~Bq}$ inhalé le sont pour une valeur du DAMA de $1 \mu \mathrm{m}$, ce qui correspond à $D_{T B}=0,08$ et $D_{p}=0,25$. II en résulte pour $\mathrm{H}_{50, \text { RESP }}^{Y}$ une valeur de $3,2 \times 10^{-4} \frac{\mathrm{Sv}}{\mathrm{Bq}}$.

En attribuant à $D_{T B}$ et $D_{p}$ les valeurs correspondant à des DAMA allant de 0,1 à $15 \mu \mathrm{m}$, les variations obtenues pour la dose engagée sont très importantes (fig. 3). Entre $0,1 \mu \mathrm{m}$, elle décroît d'un facteur 2 et entre $1 \mu \mathrm{m}$ et $15 \mu \mathrm{m}$ d'un facteur 10 .

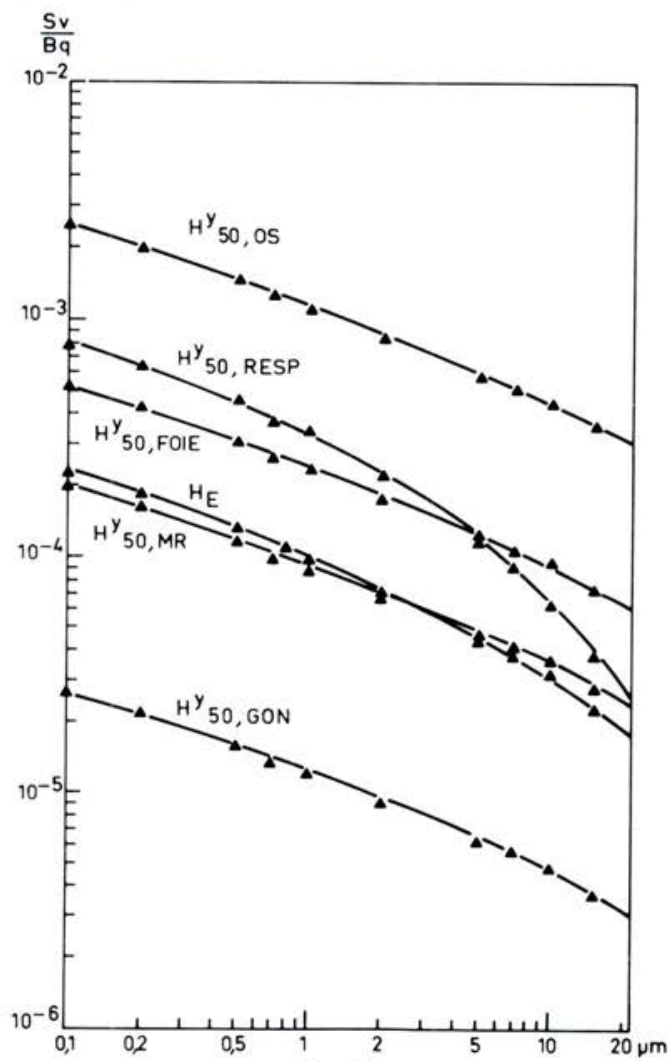

Fig. 3. 
Cette constatation montre que la connaissance de la granulométrie, souvent négligée, est au moins aussi importante que celle de la classe physico-chimique de l'aérosol.

\section{II.2. Dose engagée au squelette, à la moelle osseuse, au foie et aux gonades}

\section{II.2.1. Dépôt dans les tissus}

Les études animales et les observations humaines montrent que, dans un but de radioprotection, trois sites de dépôt du plutonium sont à prendre en considération : le foie, le squelette et les gonades. La plus grande partie de l'activité pénétrant dans le sang s'accumule dans les deux premiers tissus, mais le dépôt dans le troisième, s'il est beaucoup plus faible, est important par la dose génétique délivrée. La répartition du plutonium entre le foie et l'os est liée à la forme chimique du radionucléide. Les formes les plus transférables donnent les plus forts dépôts au niveau du squelette et les moins transférables au niveau du foie. La valeur de $45 \%$ retenue par la CIPR pour chacun des deux organes est une valeur moyenne, la fraction qui va dans le squelette se situant entre 20 et $70 \%$. A cette fraction de $45 \%$ la CIPR associe une seule longue période de rétention biologique de 100 ans pour l'os et de 40 ans pour le foie, ce qui distingue le plutonium d'autres ostéotropes dont l'uranium où l'organe comporte un compartiment à échanges rapides et un compartiment à échanges lents.

\section{II.2.2. Fonctions de rétention élémentaire}

La longueur de la période physique du plutonium permet de considérer que les rétentions effectives sont convenablement décrites par les équations de rétention biologique élémentaire suivantes :

$R_{\text {FOIE }}(t)=0,45 e^{-\frac{0,693 t}{14600}}=r_{\text {FOIE }}(t)$

$R_{\text {OS }}(t)=0,45 e^{-\frac{0,693 t}{36500}}=r_{\text {oS }}(t)$

La modélisation prenant en compte le fait que le plutonium se dépose sur les surfaces osseuses de l'endoste suppose que ce dépôt se fait uniformément au niveau des deux types de tissu osseux, compact et trabéculaire. On fait également l'hypothèse que les surfaces endostéales de ces deux tissus sont approximativement égales et que la vitesse d'élimination est la même, d'où :

$R_{\text {COMP }}(t)=R_{\text {TRAB }}(t)=0,225 e^{-\frac{0.693 t}{36500}}$

Des $10 \%$ restant de la quantité ayant pénétré dans le sang, une partie est excrétée directement, l'autre se dépose dans les tissus mous et les organes reproducteurs. Pour ces derniers, on admet un séjour illimité du plutonium, ce qui ne lie la rétention qu'à la décroissance radioactive.

$R_{G O N}(t)=3,5 \times 10^{-4} e^{-\frac{0,693 t}{8,87 \times 10^{6}}}=r_{G O N}(t)$ 


\subsubsection{Fonctions de rétention après incorporation}

\section{a) Absorption sanguine}

L'incorporation du radionucléide par inhalation conduit, pour formuler la rétention au niveau du foie, de l'os ou des gonades, à prendre en compte la cinétique d'épuration respiratoire de l'aérosol en direction du sang. Cette cinétique sera exprimée pour chacun des compartiments NP, TB et $P$ par une fonction d'absorption sanguine ayant la dimension d'un débit. Le modèle (fig. 2) montre que le passage sanguin est direct mais aussi indirect par l'absorption intestinale après remontée mucociliaire et déversement du matériel radioactif dans le tube digestif au niveau du carrefour pharyngé.

L'intervention du compartiment digestif du modèle respiratoire dans le processus d'absorption sanguine peut être représentée soit de manière globale par le facteur $f_{1}$, fraction de l'activité du radionucléide passant du tube digestif au sang, soit de manière plus détaillée à l'aide des paramètres d'un modèle digestif de type compartimental que la CIPR a établi et qui est schématisé dans la figure 2. Le paramètre d'absorption intestinale de ce modèle est une constante de transfert $\lambda_{a b}$ qui est reliée au facteur $f_{1}$ par la relation :

$f_{1}=\lambda_{a b} \int_{0}^{\infty} e^{-\left(\lambda_{i G}+\lambda_{a b}\right)^{t}} d t$

où $\lambda_{\text {ig }}$ est la constante de transfert correspondant au transit dans l'intestin grêle considéré comme le site d'absorption.

L'écriture des fonctions d'absorption sanguine en associant le modèle digestif au modèle respiratoire conduit à des expressions complexes d'un emploi malaisé. Ces expressions n'ont d'intérêt que dans le cas d'une étude métabolique, et plus particulièrement lorsque le radionucléide a une période physique courte.

Aux niveaux NP et TB où les transferts sont très rapides, il est donc légitime d'utiliser pour $\mathrm{PuO}_{2}$ des expressions simplifiées de l'absorption sanguine qui ne seront pas des débits mais des fractions, soit en associant les transferts directs et indirects :

$F_{a, b}^{N P}=D_{N P}\left(f_{a}+f_{1} f_{b}\right)$

$F_{c, d}^{T B}=D_{T B}\left(f_{c}+f_{1} f_{d}\right)$

Aux niveaux $\mathrm{P}$ et $\mathrm{L}$, par contre, où les transferts sont lents, on aura des débits d'absorption qui seront obtenus directement à partir des fonctions de rétention. Ces débits prendront une forme exponentielle simple dans le cas d'un transfert direct :

$\dot{\mathrm{F}}_{\mathrm{e}}{ }^{\mathrm{P}}(\mathrm{t})=\lambda_{\mathrm{p}} \mathrm{r}_{\mathrm{e}}{ }^{\mathrm{P}}(\mathrm{t})$

et la forme de fonctions de Bateman dans les autres cas.

Transfert indirect par $L$ :

$\dot{\mathrm{F}}_{i}^{\mathrm{P}}(\mathrm{t})=\lambda \mathrm{r}_{\mathrm{i}}^{\mathrm{L}}(\mathrm{t})$ 
Transfert indirect par le tube digestif :

$\dot{F}_{f}^{P}(t)=\lambda_{d} f_{1} r_{f}^{T B}(t)$

$\dot{F}_{g}^{P}(t)=\lambda_{d} f_{1} r_{g}^{T B}(t)$

\section{b) Rétention au niveau des organes}

Lorsque l'absorption sanguine est formulée de manière simplifiée, la rétention est obtenue de façon immédiate par le produit de la fraction transférée par la rétention élémentaire; dans le cas du foie, on a :

$$
\begin{aligned}
& r_{F O I E}^{N P}(t)=F_{a, b}^{N P} \cdot r_{F O I E}(t) \\
& r_{F O I E}^{T B}(t)=F_{c, d}^{T B} \cdot r_{F O I E}(t)
\end{aligned}
$$

Il est impossible dans le cas d'un débit d'absorption fonction du temps, d'utiliser le même processus et une opération mathématique appelée "convolution" doit être effectuée.

Soit le débit d'absorption sanguine $\dot{F}_{1}{ }^{P}(t)$ du type fonction de Bateman, on peut considérer que, sur un très petit intervalle de temps $\Delta T$, il correspond à une injection sanguine quasi instantanée.

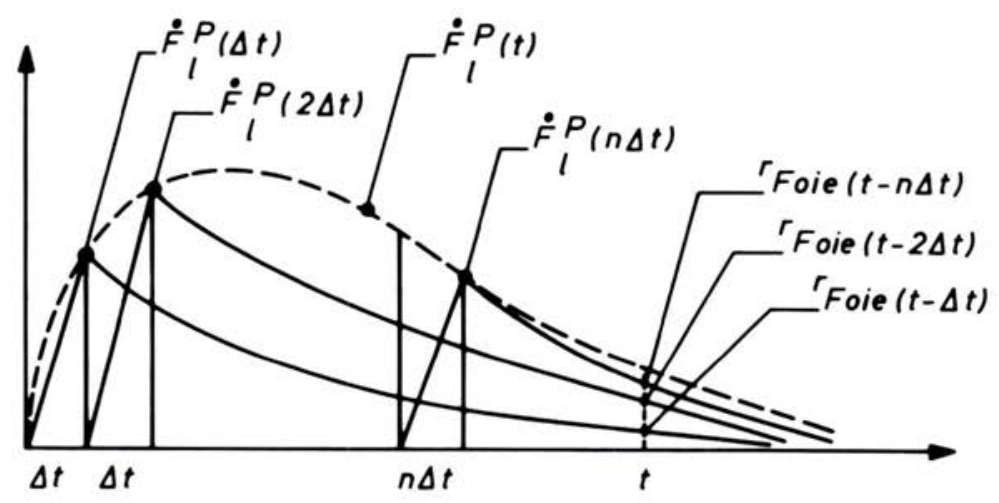

Fig. 4. 


\section{PLUTONIUM : MODÉLE DE CALCUL DES DOSES ENGAGÉES}

Le découpage $\dot{F}_{1} p(t)$ en fonction des petits intervalles de temps $\Delta t$ conduit aux petites absorptions successives :

$\dot{F}_{1}^{p}(\Delta t)=\dot{F}_{1}^{p}(0) \cdot \Delta t$

$\dot{F}_{1}^{P}(2 \Delta t)=\dot{F}_{1}^{P}(\Delta t) \cdot \Delta t$

$\dot{\mathrm{F}}_{\mathrm{P}}^{\mathrm{P}}(\mathrm{n} \Delta \mathrm{t})=\dot{\mathrm{F}}_{\mathrm{P}} \mathrm{P}(\mathrm{n}-1) \Delta \mathrm{t} . \Delta \mathrm{t}$

Chacune de ces petites absorptions entraîne une rétention élémentaire dont l'amplitude initiale est fixée par la valeur correspondante de la loi d'absorption. L'évolution de cette dernière entraîne également un décalage des rétentions élémentaires (fig. 4).

En se plaçant au temps $\mathrm{t}$, on a simultanément les rétentions :

$$
\begin{aligned}
& r_{\text {FOIE }}^{p}(t-\Delta t)=\dot{F}_{1}^{p}(\Delta t) \cdot r_{F O I E}(t-\Delta t) \\
& r_{F O I E}^{p}(t-2 \Delta t)=\dot{F}_{1}^{p}(2 \Delta t) \cdot r_{F O I E}(t-2 \Delta t)
\end{aligned}
$$$$
r_{\text {FOIE }}^{p}(t-n \Delta t)=\dot{F}_{1}^{P}(n \Delta t) \cdot r_{F O I E}(t-n \Delta t)
$$

Au total, à l'instant $t$, si $\Delta t=\frac{t}{N}$, on a :

$r_{F O I E}^{P}(t)=\sum_{n=0}^{N} \dot{F}_{1}^{P}(n \Delta t) \cdot r_{F O I E}(t-n \Delta t)$

Si maintenant, on pose $\mathrm{n} \Delta \mathrm{t}=\tau$

$\mathrm{d} \tau=(\mathrm{n}+1) \Delta \mathrm{t}-\mathrm{n} \Delta \mathrm{t}=\Delta \mathrm{t}$

et si l'on fait tendre $\mathrm{N}$ vers l'infini pour $\mathrm{t}$ fixé, $\mathrm{d} \tau \rightarrow \mathrm{o}$. La sommation conduit alors à l'intégrale de convolution :

$r_{\text {FOIE }}^{P, I}(t)=\int_{0}^{t} \dot{F}_{1}^{P}(\tau) \cdot r_{F O I E}(t-\tau) d \tau$

qui est la rétention après incorporation recherchée. Pour l'ensemble des transferts dont l'origine est au niveau $\mathrm{P}$ :

$\mathrm{r}_{\mathrm{FOIE}}^{\mathrm{P}}(\mathrm{t})=\sum_{1} \int_{0}^{\mathrm{t}} \dot{\mathrm{F}}_{1}^{\mathrm{P}}(\tau) \cdot \mathrm{r}_{\mathrm{FOIE}}(\mathrm{t}-\tau) \mathrm{d} \tau$

\section{II.2.4. Doses engagées}

\section{a) Nombre total de transformations dans l'organe}

En conservant l'exemple du foie, ce nombre sera obtenu par l'intégration sur 50 ans des fonctions de rétention après incorporation, soit la somme :

$U_{50, \mathrm{FOIE}}^{Y}=U_{50, \mathrm{FOIE}}^{\mathrm{NP}}+U_{50, \mathrm{FOIE}}^{\mathrm{TB}}=U_{50, \mathrm{FOIE}}^{\mathrm{P}}$ 
Pour les deux premiers termes de la somme, le calcul est simple :

$U_{50, F O I E}^{N P}=F_{a, b}^{N P} \cdot K \cdot \int_{0}^{t} r_{F O I E}(t) d t$

$U_{50, F O I E}^{T B}=F_{c, d}^{T B} \cdot K \cdot \int_{0}^{T} r_{F O I E}(t) d t$

Pour le dernier terme où intervient la convolution, le calcul est compliqué par la nécessité d'une double intégration:

on a en effet :

$\mathrm{U}_{50, \text { FOIE }}^{\mathrm{P}}=\int_{0}^{T} \sum_{1} \int_{0}^{\mathrm{t}} \dot{\mathrm{F}}_{1}^{\mathrm{P}}(\tau) \cdot \mathrm{r}_{\mathrm{FOIE}}(\mathrm{t}-\tau) \mathrm{d} \tau \mathrm{dt}$.

Cependant, comme le temps $T$ est suffisamment grand comparé aux temps de résidence dans le poumon, l'intégrale peut être dédoublée et le débit d'absorption sanguine intégré séparément, d'où :

$\mathrm{U}_{50, \text { FOIE }}^{\mathrm{P}}=\sum_{1} \int_{0}^{\infty} \dot{\mathrm{F}}_{1}^{\mathrm{P}}(\mathrm{t}) \mathrm{dt} \cdot\left[\int_{0}^{T r_{F O I E}}(\mathrm{t}) \mathrm{dt}\right]$

\section{b) Dose engagée au foie et aux gonades}

L'expression de la dose engagée au foie et celle de la dose engagée aux gonades ont la même structure qui est celle obtenue pour la dose engagée à l'appareil respiratoire. En ce qui concerne le facteur dosimétrique, la seule différence intervient au niveau de l'énergie effective où la masse est celle de l'organe considéré : $M_{F O I E}$ de $1800 \mathrm{~g}$ et $M_{G O N}$ de $35 \mathrm{~g}$. On a donc :

$\mathrm{H}_{50, \text { FOIE }}^{\Upsilon}=S\left(\varepsilon_{\mathrm{FOIE}}\right) \cdot U_{50, \text { FOIE }}^{\curlyvee}$

$\mathrm{H}_{50, \mathrm{GON}}^{Y}=\mathrm{S}\left(\varepsilon_{\mathrm{GON}}\right) \cdot U_{50, \mathrm{GON}}^{Y}$

\section{c) Dose engagée aux surfaces endostéales (SE)}

Le tissu radiosensible au niveau des surfaces osseuses de l'endoste est constitué par les cellules endostéales qui sont l'un des éléments actifs du renouvellement osseux. Le tissu émetteur de rayonnements est le couple os compact-os trabéculaire et le nombre total de transformations à ce niveau est donné par:

$u_{50 . \text { COMP }}=U_{50 . \text { TRAB }}=K \cdot \int_{0}^{T} 0,2250 e^{-\frac{0,693 t}{36500}} d t$

La dose engagée répond à l'expression :

$\mathrm{H}_{50, \text { OS }}^{\Upsilon}=\mathrm{S}\left(\boldsymbol{\varepsilon}_{\text {COMP }}\right) . U_{50, \text { COMP }}^{\Upsilon}+\mathrm{S}\left(\boldsymbol{\varepsilon}_{\text {TRAB }}\right) \cdot \cup_{50, \text { TRAB }}^{\Upsilon}$ 
où: $\mathrm{S}\left(\varepsilon_{\mathrm{cOMP}}\right)=\mathrm{k} \cdot \boldsymbol{\varepsilon}(\mathrm{SE}-\mathrm{COMP})_{\mathrm{a}}$

$$
\mathrm{S}\left(\boldsymbol{\varepsilon}_{\mathrm{TRAB}}\right)=\mathrm{k} \cdot \boldsymbol{\varepsilon}(\mathrm{SE}-\mathrm{TRAB})_{\alpha}
$$

La fraction absorbée de l'énergie des deux tissus sources dans le tissu cible SE est la même :

$\mathrm{FA}(\mathrm{SE}-\mathrm{COMP})_{\alpha}=\mathrm{FA}(\mathrm{SE}-\mathrm{TRAB})_{\alpha}=0,25$

la masse du tissu cible est $\mathrm{M}_{\mathrm{SE}}=120 \mathrm{~g}$.

\section{d) Dose engagée à la moelle osseuse rouge (MR)}

La moelle osseuse rouge qui contient les cellules souches des lignées cellulaires sanguines est le second tissu radiosensible du squelette. Sa localisation limitée à l'os trabéculaire conduit à écrire :

$\mathrm{H}_{50, \mathrm{MR}}^{\curlyvee}=\mathrm{S}\left(\boldsymbol{\varepsilon}_{\mathrm{MR}}\right) \mathrm{U}_{50, \text { TRAB }}^{\Upsilon}$ où $\mathrm{S}\left(\varepsilon_{\mathrm{MR}}\right)=\mathrm{k} \cdot \boldsymbol{\varepsilon}(\mathrm{MR} \leftarrow \mathrm{TRAB})_{\alpha}$

La fraction absorbée de l'énergie du tissu source trabéculaire dans le tissu cible MR est égale à :

$\mathrm{FA}(\mathrm{MR} \leftarrow \mathrm{TRAB})_{\alpha}=0,50$, et la masse du tissu cible $\mathrm{M}_{\mathrm{MR}}=1500 \mathrm{~g}$

\section{e) Importance de la granulométrie}

Comme dans le cas de l'appareil respiratoire, les doses engagées aux organes précédents vont varier de manière importante avec la taille particulaire de l'aérosol. Cette variation s'étend de $2,45 \times 10^{-3} \frac{\mathrm{Sv}}{\mathrm{Bq}}$ à $3,37 \times 10^{-4} \frac{\mathrm{Sv}}{\mathrm{Bq}}$ entre 0,1 et $15 \mu \mathrm{m}$ pour les surfaces endostéales qui sont le tissu le plus exposé (fig. 3). Les courbes présentent un parallélisme qui s'explique par l'application des mêmes facteurs granulométriques aux fonctions d'absorption sanguine qui sont communes à tous les organes. Le comportement différent de la courbe relative à l'appareil respiratoire tient à la nature différente de sa rétention.

\section{II.3. Dose engagée effective}

L'application aux doses engagées obtenues du coefficient $W_{T}$ représentant la proportion du risque d'effet stochastique propre à chaque organe ou tissu T conduit à dresser le tableau suivant, le DAMA étant de $1 \mu \mathrm{m}$ :

\begin{tabular}{|c|c|c|c|c|c|}
\hline $\mathrm{T}$ & $\mathrm{H}_{50, \mathrm{~T}}$ & $\begin{array}{l}\text { Sv } \\
\mathrm{Bq}\end{array}$ & $W_{T}$ & $H_{50, T}^{Y}$ & $\frac{\mathrm{Sv}}{\mathrm{Bq}}$ \\
\hline $\begin{array}{l}\text { RESP } \\
\text { OS } \\
\text { MR } \\
\text { FOIE } \\
\text { GON }\end{array}$ & $\begin{array}{l}3,2 x \\
9,7 \times \\
7,7 \times \\
2,1 \times x \\
1,2 x\end{array}$ & $\begin{array}{l}10^{-4} \\
10^{-4} \\
10^{-5} \\
10^{-4} \\
10^{-5}\end{array}$ & $\begin{array}{l}3,9 \\
2,9 \\
9,3 \\
1,2 \\
2,8\end{array}$ & $\begin{array}{l}x \\
x \\
x \\
x \\
x\end{array}$ & $\begin{array}{l}10^{-5} \\
10^{-5} \\
10^{-6} \\
10^{-5} \\
10^{-6}\end{array}$ \\
\hline
\end{tabular}


Le tissu qui reçoit la dose engagée non pondérée par le coefficient de risque la plus élevée est l'os (surfaces endostéales). La dose engagée pondérée la plus élevée est délivrée à l'appareil respiratoire. Des autres tissus, seuls, l'os, la moelle osseuse et le foie reçoivent une dose engagée pondérée égale ou supérieure à $10 \%$ de celle du tissu respiratoire. Ce sont ces 3 tissus plus l'appareil respiratoire qui vont fournir la dose engagée effective par la somme :

$\Sigma_{\mathrm{T}} \mathrm{W}_{\mathrm{T}} \mathrm{H}_{50, \mathrm{~T}}^{\mathrm{Y}}=\mathrm{H}_{\mathrm{E}}=8,9 \times 10^{-5} \frac{\mathrm{Sv}}{\mathrm{Bq}}$

La dose engagée effective est également influencée par le granulométrie comme le montre la figure 3 . Pour une granulométrie de $0,1 \mu \mathrm{m}$ elle est de $2,2 \times 10^{-4} \frac{\mathrm{Sv}}{\mathrm{Bq}}$ et elle passe à $2,2 \times 10^{-5} \frac{\mathrm{Sv}}{\mathrm{Bq}}$ pour une granulométrie de $15 \mu \mathrm{m}$.

L'emploi d'un modèle osseux, dans lequel le plutonium au lieu de rester au niveau des surfaces endostéales des tissus compact et trabéculaire subit un certain degré d'enfouissement, conduit à une valeur plus faible de la dose engagée à l'endoste et à une augmentation de la dose engagée à la moelle rouge. On obtient le tableau suivant, toujours pour un DAMA de $1 \mu \mathrm{m}$.

\begin{tabular}{|c|c|c|c|c|c|}
\hline $\mathrm{T}$ & $H_{50, T}^{Y}$ & $\begin{array}{c}\mathrm{Sv} \\
\mathrm{Bq}\end{array}$ & $W_{T}$ & $H_{50, T}^{Y}$ & $\frac{\mathrm{Sv}}{\mathrm{Bq}}$ \\
\hline $\begin{array}{l}\text { RESP } \\
\text { OS } \\
\text { MR } \\
\text { FOIE }\end{array}$ & $\begin{array}{l}3,2 x \\
3,1 \times \\
9,2 x \\
2,1 \times\end{array}$ & $\begin{array}{l}10^{-4} \\
10^{-5} \\
10^{-5} \\
10^{-4}\end{array}$ & $\begin{array}{l}3,9 \\
9,2 \\
1,1 \\
1,2\end{array}$ & $\begin{array}{l}x \\
x \\
x \\
x\end{array}$ & $\begin{array}{l}10^{-5} \\
10^{-7} \\
10^{-5} \\
10^{-5}\end{array}$ \\
\hline
\end{tabular}

La dose engagée effective prend alors la valeur de $6,2 \times 10^{-5} \frac{\mathrm{Sv}}{\mathrm{Bq}}$ légèrement plus faible que la précédente.

\section{CONCLUSION}

Le calcul des doses engagées dépend, au plan métabolique, d'une part de modèles anatomophysiologiques généraux (modèle respiratoire, modèle digestif), d'autre part de modèles de rétention élémentaire propres à chaque nucléide. Les valeurs appliquées aux paramètres de ces modèles sont des valeurs moyennes déduites d'études expérimentales et de quelques observations humaines. II en résulte des incertitudes qui peuvent être importantes. C'est le cas des valeurs attribuées aux paramètres décrivant le comportement métabolique à très long terme de radionucléide comme le plutonium. Au plan dosimétrique où les grandeurs utilisées paraissent à première vue plus accessibles à la mesure, on rencontre, en fait, les mêmes difficultés pour le modèle osseux et les fractions absorbées. Enfin, les coefficients de risque utilisés pour le calcul de la dose engagée effective comportent également une bonne part d'incertitude. 
Les valeurs de doses engagées que l'on obtient par les calculs précédents ne sont donc pas des chiffres précis, mais des valeurs guides permettant la dérivation de limites basées sur des hypothèses raisonnables. Ces hypothèses ne sont pas définitives et l'on voit combien une granulométrie différente de $1 \mu \mathrm{m}$ et l'adoption d'un modèle osseux autre que le dépôt du plutonium strictement en surface changent les résultats.

\section{BIBLIOGRAPHIE}

[1] Commission internationale de protection radiologique. - Limits for intakes of radionuclides by workers. ICRP Publication 30. Part I and supplement to Part I. Oxford: Pergamon press, 1979.

[2] Communautés européennes. - Directive du Conseil du 15 juillet 1980 portant modification des directives fixant les normes de base relatives à la protection sanitaire de la population et des travailleurs contre les dangers des rayonnements ionisants. J. Off. Comm. Europ, 17 septembre 1980, n L 246.

[3] MENOUX B., BEAU P.G. - Evaluation des équivalents de dose délivrés par les principaux radionucléides impliqués dans le fonctionnement des installations nucléaires. Rapport DPr - 82/3 - SPS, 1982.

[4] NENOT J.C., STATHER J.W. - Toxicité du plutonium, de l'américium et du curium. Paris: Technique et documentation, 1978.

[5] PRIEST N.D., HUNT B.W. - The calculation of ALIs for plutonium-239 in man using a bone model which allows for plutonium burial and recycling. Phys. Med. Biol. 1979, 24, 525-546. 